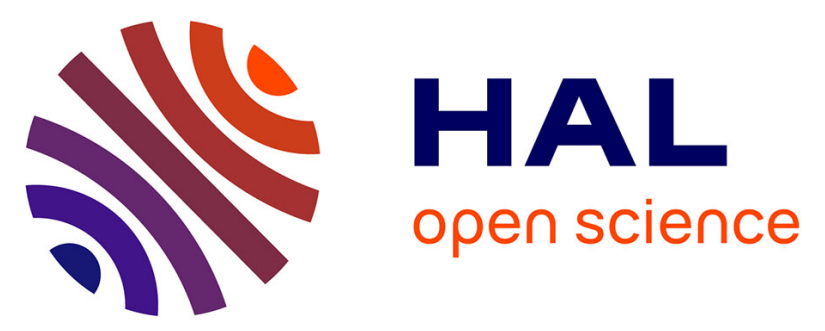

\title{
Stabilization with imprecise measurements: application to a vision based landing problem
}

\author{
Frédéric Mazenc, Laurent Burlion, Victor Gibert
}

\section{To cite this version:}

Frédéric Mazenc, Laurent Burlion, Victor Gibert. Stabilization with imprecise measurements: application to a vision based landing problem. ACC 2018 - American Control Conference, Jun 2018, Milwaukee, United States. 10.23919/acc.2018.8431402 . hal-01848888

\section{HAL Id: hal-01848888 \\ https://hal.inria.fr/hal-01848888}

Submitted on 25 Jul 2018

HAL is a multi-disciplinary open access archive for the deposit and dissemination of scientific research documents, whether they are published or not. The documents may come from teaching and research institutions in France or abroad, or from public or private research centers.
L'archive ouverte pluridisciplinaire HAL, est destinée au dépôt et à la diffusion de documents scientifiques de niveau recherche, publiés ou non, émanant des établissements d'enseignement et de recherche français ou étrangers, des laboratoires publics ou privés. 


\title{
Stabilization with imprecise measurements: application to a vision based landing problem
}

\author{
Frédéric Mazenc Laurent Burlion Victor Gibert
}

\begin{abstract}
The problem of stabilizing a nonlinear system approximated in a neighborhood of the origin by a saturated chain of integrators when the variables are not accurately measured is addressed. A recent backstepping approach with delay is adapted to the specificity of the studied system. The results are used to solve a control problem that arises in the context of vision based landing of a civil aircraft.

Index Terms-Stabilization, delay, backstepping, bounded control, vision-based control.
\end{abstract}

\section{INTRODUCTION}

The difficult problem of stabilizing systems with bounded control has a long history. To solve it, several techniques of control design have been developed for several families of systems. Semi-global state and output feedback stabilization results have been obtained via linear control laws acting inside a saturation function, as done for instance in the contribution [13]. Crucial regional stability results for families of linear and nonlinear systems, which rely in particular on LMI techniques, are presented in the research monograph [15]. The techniques called the forwarding and the bounded backstepping provide with globally asymptotically stabilizing control laws for some families of nonlinear systems. Bounded backstepping has been developed in particular in the contributions [2] and [7] and forwarding in many contributions, notably in [16], [11] and [14].

Recently, a significantly different backstepping design has been proposed in the papers [8] and [9]. It relies on the introduction in the expression of the control laws of artificial pointwise delays which circumvent the problem of determining Lie derivatives of the fictitious control laws used in the classical approach. Thus, it makes it possible to relax the smoothness requirement which is imposed on the fictitious control in all the previous contributions on backstepping. Moreover, for many systems of feedback or feedforward form, it can be adapted to the problem of determining stabilizing bounded feedbacks and leads to analytic expressions that are simpler than those of the feedbacks constructed in [2] and [7]. Finally, by contrast with other techniques, global stability results are obtained via this approach.

Mazenc is with Inria, University Paris-Saclay, L2S (CNRS) CentraleSupélec, Gif-sur-Yvette, France. frederic.mazenc (at) 12s.centralesupelec.fr

Burlion is with the Department of Information Processing and Systems, ONERA - The French Aerospace Lab, Toulouse, France. Burlion was partly supported by ANR VISIOLAND project ANR-13-CORD-0012 dedicated to VISIOn based aircraft LANDing techniques. laurent.burlion (at) onera.fr

Gibert is with Airbus Operations, S.A.S, Toulouse, France. victor.gibert (at) airbus.com
The advantages of the approach of [8] led us in the present work to adapt it to a specific control design problem motivated by an engineering application. Indeed, we here interest us to the emergency landing of a civil aircraft : the runway being unequipped, the aircraft must rely an embedded video camera which, from the control point of view, poses the problem of controlling a perspective dynamical system [5]. In the present paper, we focus on the lateral motion and develop a new control law for a specific three dimensional feedforward system. Due to vision, the key difficulty presented by the model, is that only imprecise measurements of the two first state variables are available. As a consequence, we cannot directly apply results providing with semi-global or regional stability mentioned above, nor the design proposed in [8] or its extension [9]. Thus, we propose a new theoretical control design technique, which extends the theory presented in [8] and [9]. This theoretical result is new but owes a great deal to the forwarding theory [16], [10]. The control laws we construct are bounded and result in a globally asymptotically stable closed-loop system. An important part of our work is devoted to the assessment of the control design through numerical simulations based on real data.

The paper is organized as follows. The problem is presented in Section II. Technical lemmas are stated in Section III. The main result is in Section IV. The main result is illustrated in the specific context of vision based landing of a civil aircraft in Section V. Concluding remarks in Section VI end the paper.

\section{A. Notation}

The notation will be simplified whenever no confusion can arise from the context. The Euclidean norm in $\mathbb{R}^{a}$, and the induced norm of matrices, are denoted by $|\cdot|$. Given any constant $T>0$, we let $C_{\text {in }}$ denote the set of all continuous functions $\phi:[-T, 0] \rightarrow \mathbb{R}^{a}$, which we call the set of all initial functions. We define $\Xi_{t} \in C_{\text {in }}$ by $\Xi_{t}(s)=\Xi(t+s)$ for all choices of $\Xi, s \leq 0$, and $t \geq 0$ for which the equality is defined.

Given $L>0$, sat $t_{L}$ denotes the classical symmetric saturation function i. e.

$$
\begin{gathered}
\operatorname{sat}_{L}(x)=\max (-L, \min (L, x)), \quad \forall x \in \mathbb{R} . \\
\text { II. PRoBlem STATEMENT }
\end{gathered}
$$

We consider the system

$$
\left\{\begin{array}{l}
\dot{x}_{1}=\operatorname{sat}_{L_{1}}\left(x_{2}\right) \\
\dot{x}_{2}=\operatorname{sat}_{L_{2}}\left(x_{3}\right) \\
\dot{x}_{3}=\operatorname{sat}_{L_{3}}(u),
\end{array}\right.
$$


where $\left(x_{1}, x_{2}, x_{3}\right) \in \mathbb{R}^{3}, u \in \mathbb{R}$ is the input and $L_{i}>0, i=$ $1,2,3$.

We assume that the outputs are:

$$
\begin{aligned}
& y_{1}(t)=\eta(t) x_{1}(t)+\delta_{1}(t) \\
& y_{2}(t)=x_{2}(t)+\delta_{2}(t) \\
& y_{3}(t)=x_{3}(t)
\end{aligned}
$$

where $\delta_{1}, \delta_{2}$ and $\eta$ are unknown but piecewise-continuous functions for which there are constants $\underline{\eta}>0, \bar{\eta}>\underline{\eta}, \bar{\delta}_{1} \geq 0$ and $\bar{\delta}_{2} \geq 0$ such that

$$
\eta(t) \in[\underline{\eta}, \bar{\eta}] \quad, \quad\left|\delta_{i}(t)\right| \leq \bar{\delta}_{i} \quad, \quad i=1,2
$$

for all $t \geq 0$.

The objective of this paper is the global robust stabilization of the origin of the system (2) through a bounded feedback using only the measurements $y_{i}$ in (3).

The fact that $\eta(t)$ is present in $y_{1}$ is a serious difficulty. It implies that classical approaches do not apply. Although the system (2) is a feedforward system (see for instance [11] for the definition of feedforward system), the forwarding approach (notably the results of [11] and [16]) does not apply, except under an additional assumption pertaining to the size of $\bar{\eta}-\eta$. But for practical reasons, we cannot impose an assumption of this type.

Since (2) is not in feedback form, classical backstepping results (see for instance [14]) do not apply. Since the function $\eta$ is unknown, not necessarily differentiable and with a possibly unbounded first derivative, bounded backstepping results in [7], [6] do not apply. Other approaches (see for instance [10]) do not apply too. Finally, let us observe that one cannot directly apply the result of [8], which does not take into account the case where there are uncertainties on the measurements. Thus, to the best of our knowledge, the problem we solve is open.

\section{TECHNICAL PRELIMINARY RESULTS}

\section{A. Stability of a system with a distributed delay}

The following lemma will have a fundamental role when we establish the main result of our paper.

Lemma 1: Consider the system

$$
\dot{\xi}=\alpha\left(t, \xi_{t}\right)+d(t)
$$

where $\xi \in \mathbb{R}, d:[0,+\infty) \rightarrow \mathbb{R}$ is a piecewise continuous function such that $|d(t)| \leq \bar{d}$ for all $t \geq 0$,

$$
\alpha\left(t, \xi_{t}\right)=-\mathfrak{s} \int_{t-\tau}^{t} e^{q(\ell-t)} \int_{\ell-\tau}^{\ell} e^{r(m-\ell)} \phi(\eta(m) \xi(m)) d m d \ell
$$

where the constant $\mathfrak{s}$ is defined by:

$$
\mathfrak{s}=\frac{q r}{\left(1-e^{-q \tau}\right)\left(1-e^{-r \tau}\right)},
$$

with $\tau>0 q>0, r>0$ and where $\phi: \mathbb{R} \rightarrow \mathbb{R}$ is (i) odd, (ii) non-decreasing, (iii) globally Lipschitz, with Lipschitz constant $\phi_{c}>0$ and such that there are positive constants $\phi_{s}$ and $\bar{\phi}$ such that

$$
\lim _{s \rightarrow+\infty} \phi(s)=\bar{\phi} \quad, \quad \phi_{s} s \leq \phi(s) \quad, \quad \forall s \in\left[0, \frac{\bar{\phi}}{2}\right] .
$$

Moreover,

$$
8 \tau \phi_{c} \bar{\eta}(\bar{\phi}+\bar{d})+4 \bar{d} \leq \bar{\phi}
$$

and

$$
\tau \mathfrak{p}<\mathfrak{c}
$$

with

$$
\mathfrak{p}=\phi_{c}^{2} \bar{\eta}^{2} \quad \text { and } \quad \mathfrak{c}=\inf _{a \in \mathfrak{E}} \frac{\phi(\underline{\eta}|a|)}{3|a|}
$$

where

$$
\mathfrak{E}=\left\{b \in \mathbb{R}: 4 \tau \phi_{c} \bar{\eta}(\bar{\phi}+\bar{d})+2 \bar{d} \geq \phi(\underline{\eta}|b|)\right\} .
$$

Then there is $t_{\sharp} \geq 4 \tau$ such that for all $t \geq t_{\sharp}$,

$$
|\xi(t)| \leq \frac{\left(2 \phi_{c} \bar{\eta} \tau+1\right) \bar{d}}{\sqrt{2 \mathfrak{c}(\mathfrak{c}-\tau \mathfrak{p})}}
$$

Remark. 1. The system (5) cannot be Input-to-State-Stable with respect to $d$ because $\alpha$ is bounded. 2. The inequality (9) ensures that there is $\mathfrak{k}>0$ such that $\mathfrak{E}=[-\mathfrak{k}, \mathfrak{k}]$. 3. The relations in (8) ensure that $\mathfrak{c}>0$.

Proof. First, notice that finite escape time phenomenon does not occur for (5) because the function $\phi$ is bounded. Second, consider the positive definite quadratic function

$$
V(\xi)=\frac{1}{2} \xi^{2}
$$

and let us define for later use the function

$$
W(\xi)=\xi \phi(\underline{\eta} \xi) .
$$

Simple calculations give

$$
\begin{aligned}
\dot{V}(t)= & -\mathfrak{s} \xi(t) \int_{t-\tau}^{t} e^{q(\ell-t)} \int_{\ell-\tau}^{\ell} e^{r(m-\ell)} \phi(\eta(m) \xi(t)) d m d \ell \\
& +\mathfrak{s} \xi(t) \int_{t-\tau}^{t} e^{q(\ell-t)} \int_{\ell-\tau}^{\ell} e^{r(m-\ell)}[\phi(\eta(m) \xi(t)) \\
& -\phi(\eta(m) \xi(m))] d m d \ell+\xi(t) d(t) \\
\leq & -\mathfrak{s} \xi(t) \int_{t-\tau}^{t} e^{q(\ell-t)} \int_{\ell-\tau}^{\ell} e^{r(m-\ell)} \phi(\eta(m) \xi(t)) d m d \ell \\
& +\mathfrak{s} \phi_{c}|\xi(t)| \int_{t-\tau}^{t} e^{q(\ell-t)} \int_{\ell-\tau}^{\ell} e^{r(m-\ell)}|\eta(m)| \\
& \times|\xi(t)-\xi(m)| d m d \ell+\xi(t) d(t),
\end{aligned}
$$

where the last inequality is a consequence of the fact that $\phi$ is Lipschitz with Lipschitz constant $\phi_{c}$. From the other properties of $\phi$ and the fact that, for all $t \geq 0$, $\mathfrak{s} \int_{t-\tau}^{t} e^{q(\ell-t)} \int_{\ell-\tau}^{\ell} e^{r(m-\ell)} d m d \ell=1$, we deduce successively

$$
\begin{aligned}
\dot{V}(t) \leq & -\mathfrak{s} \xi(t) \int_{t-\tau}^{t} e^{q(\ell-t)} \int_{\ell-\tau}^{\ell} e^{r(m-\ell)} \phi(\underline{\eta} \xi(t)) d m d \ell \\
& +\mathfrak{s} \phi_{c}|\xi(t)| \int_{t-\tau}^{t} e^{q(\ell-t)} \int_{\ell-\tau}^{\ell} e^{r(m-\ell)} \bar{\eta} \\
& \times|\xi(t)-\xi(m)| d m d \ell+\xi(t) d(t) \\
= & -W(\xi(t))+\mathfrak{s} \phi_{c} \bar{\eta}|\xi(t)| \int_{t-\tau}^{t} e^{q(\ell-t)} \int_{\ell-\tau}^{\ell} e^{r(m-\ell)} \\
& \times|\xi(t)-\xi(m)| d m d \ell+\xi(t) d(t) .
\end{aligned}
$$


Now, observe that for all $m \in \mathbb{R}, t \in \mathbb{R}$ such that $0 \leq m \leq t$, the inequality

$$
|\xi(m)-\xi(t)| \leq \int_{m}^{t}|\dot{\xi}(s)| d s
$$

is satisfied. It follows that for all $t \geq 2 \tau$,

$$
\begin{aligned}
\dot{V}(t) \leq & -W(\xi(t))+\mathfrak{s} \phi_{c} \bar{\eta}|\xi(t)| \int_{t-\tau}^{t} e^{q(\ell-t)} \int_{\ell-\tau}^{\ell} e^{r(m-\ell)} \\
& \times \int_{m}^{t}|\dot{\xi}(s)| d s d m d \ell+\xi(t) d(t) \\
\leq & -W(\xi(t))+\phi_{c} \bar{\eta}|\xi(t)| \int_{t-2 \tau}^{t}|\dot{\xi}(s)| d s+\xi(t) d(t),
\end{aligned}
$$

where the last inequality is a consequence of the fact that $\int_{m}^{t}|\dot{\xi}(s)| d s \leq \int_{t-2 \tau}^{t}|\dot{\xi}(s)| d s$ for all $m \in[t-2 \tau, t]$. Now, observe that

$$
\begin{aligned}
|\dot{\xi}(t)| \leq & \mathfrak{s} \int_{t-\tau}^{t} e^{q(\ell-t)} \int_{\ell-\tau}^{\ell} e^{r(m-\ell)} \phi(\bar{\eta}|\xi(m)|) d m d \ell \\
& +|d(t)| \\
\leq & \bar{\phi}+\bar{d}
\end{aligned}
$$

Combining the last inequality with (19), we obtain

$$
\begin{aligned}
\dot{V}(t) \leq & -W(\xi(t))+\left[2 \tau \phi_{c} \bar{\eta}(\bar{\phi}+\bar{d})+\bar{d}\right]|\xi(t)| \\
= & -\frac{1}{2} W(\xi(t)) \\
& +\left[2 \tau \phi_{c} \bar{\eta}(\bar{\phi}+\bar{d})+\bar{d}-\frac{1}{2} \phi(\underline{\eta}|\xi(t)|)\right]|\xi(t)| .
\end{aligned}
$$

Therefore when

$$
4 \tau \phi_{c} \bar{\eta}(\bar{\phi}+\bar{d})+2 \bar{d} \leq \phi(\underline{\eta}|\xi(t)|)
$$

then

$$
\dot{V}(t) \leq-\frac{1}{2} W(\xi(t))
$$

Since $W$ is positive definite, we deduce there is $t_{0} \geq 4 \tau$ such that for all $t \geq t_{0},|\xi(t)| \in \mathfrak{E}$, where $\mathfrak{E}$ is the set defined in (12). Then we deduce from (19) and the definition of $\mathfrak{c}$ in (11) that

$$
\dot{V}(t) \leq-3 \mathfrak{c} \xi(t)^{2}+\phi_{c} \bar{\eta}|\xi(t)| \int_{t-2 \tau}^{t}|\dot{\xi}(s)| d s+\xi(t) d(t)
$$

and

$$
|\dot{\xi}(t)| \leq \phi_{c} \bar{\eta} \mathfrak{s} \int_{t-\tau}^{t} e^{q(\ell-t)} \int_{\ell-\tau}^{\ell} e^{r(m-\ell)}|\xi(m)| d m d \ell+|d(t)|
$$

for all $t \geq t_{0}$. We deduce that

$$
\begin{aligned}
\dot{V}(t) \leq & -3 \mathfrak{c} \xi(t)^{2}+\phi_{c} \bar{\eta}|\xi(t)| \int_{t-2 \tau}^{t}\left[\phi_{c} \bar{\eta} \mathfrak{s} \int_{s-\tau}^{s} e^{q(\ell-s)}\right. \\
& \left.\times \int_{\ell-\tau}^{\ell} e^{r(m-\ell)}|\xi(m)| d m d \ell+|d(s)|\right] d s \\
& +\xi(t) d(t) \\
\leq & -3 \mathfrak{c} \xi(t)^{2}+\mathfrak{p s} \int_{t-2 \tau}^{t} \int_{s-\tau}^{s} e^{q(\ell-s)} \\
& \times \int_{\ell-\tau}^{\ell} e^{r(m-\ell)}|\xi(t)||\xi(m)| d m d \ell d s+\Omega(t)|\xi(t)|
\end{aligned}
$$

with $\mathfrak{p}$ defined in (11) and

$$
\Omega(t)=\phi_{c} \bar{\eta} \int_{t-2 \tau}^{t}|d(s)| d s+|d(t)| .
$$

Using $|\xi(t)||\xi(m)| \leq \frac{1}{2} \xi(t)^{2}+\frac{1}{2} \xi(m)^{2}$, we obtain

$$
\begin{aligned}
\dot{V}(t) \leq & {\left[-3 \mathfrak{c}+\tau \phi_{c}^{2} \bar{\eta}^{2}\right] \xi(t)^{2}+\frac{1}{2} \mathfrak{p s} \int_{t-2 \tau}^{t} \int_{s-\tau}^{s} e^{q(\ell-s)} } \\
& \times \int_{\ell-\tau}^{\ell} e^{r(m-\ell)} \xi(m)^{2} d m d \ell d s+\Omega(t)|\xi(t)| \\
\leq & -2 \mathfrak{c} \xi(t)^{2}+\frac{\mathfrak{p s}}{2} \int_{t-2 \tau}^{t} \int_{s-\tau}^{s} e^{q(\ell-s)} \\
& \times \int_{\ell-\tau}^{\ell} e^{r(m-\ell)} \xi(m)^{2} d m d \ell d s+\Omega(t)|\xi(t)|
\end{aligned}
$$

where the last inequality is a consequence of (10). Then, using the inequality $\Omega(t)|\xi(t)| \leq \mathfrak{c} \xi(t)^{2}+\frac{1}{4 \mathfrak{c}} \Omega(t)^{2}$, we obtain

$$
\begin{aligned}
\dot{V}(t) \leq & -\mathfrak{c} \xi(t)^{2}+\frac{\mathfrak{p s}}{2} \int_{t-2 \tau}^{t} \int_{s-\tau}^{s} e^{q(\ell-s)} \\
& \times \int_{\ell-\tau}^{\ell} e^{r(m-\ell)} \xi(m)^{2} d m d \ell d s+\frac{1}{4 \mathfrak{c}} \Omega(t)^{2} .
\end{aligned}
$$

Since $\Omega(t) \leq\left(2 \phi_{c} \bar{\eta} \tau+1\right) \bar{d}$, we deduce that

$$
\begin{aligned}
\dot{V}(t) \leq & -2 \mathfrak{c} V(\xi(t))+2 \tau \mathfrak{p} \sup _{a \in[t-4 \tau, t]} V(\xi(a)) \\
& +\frac{\left(2 \phi_{c} \bar{\eta} \tau+1\right)^{2} \bar{d}^{2}}{4 \mathfrak{c}}
\end{aligned}
$$

Then (10) allows us to conclude.

\section{B. Robust stabilization of a system}

In this section, we establish a result of robust stabilization for the system

$$
\left\{\begin{array}{l}
\dot{\lambda}_{1}=\operatorname{sat}_{U_{1}}\left(\lambda_{2}\right) \\
\dot{\lambda}_{2}=\operatorname{sat}_{U_{2}}(\rho)
\end{array}\right.
$$

with $\left(\lambda_{1}, \lambda_{2}\right) \in \mathbb{R}^{2}, U_{1}>0$ and $U_{2}>0$ and where $\rho$ is the input. This result draws its inspiration from [16] and [10].

Let $q, r, \bar{v}$ and $\bar{w}$ be positive constants satisfying

$$
\bar{v}<\min \left\{\frac{U_{2}}{2}, q U_{1}\right\}, \quad \bar{w} \leq \frac{\bar{v}}{3} .
$$

Lemma 2: Let us consider the system (31) with

$$
\rho(t)=-q \lambda_{2}(t)+s a t_{\bar{v}}\left(-r q \lambda_{1}(t)-r \lambda_{2}(t)+w(t)\right),
$$

where $w$ is a function such that, for all $t \geq 0$,

$$
|w(t)| \leq \bar{w} .
$$

Let $\lambda_{3}=\lambda_{2}+q \lambda_{1}$. Then there is $t_{*} \geq 0$ such that for all $t \geq t_{*}$,

$$
\left\{\begin{array}{l}
\dot{\lambda}_{1}=-q \lambda_{1}+\lambda_{3} \\
\dot{\lambda}_{3}=-r \lambda_{3}+w .
\end{array}\right.
$$

Proof. To ease the proof, let us introduce the simplifying notation

$$
v(t)=\operatorname{sat}_{\bar{v}}\left(-r q \lambda_{1}(t)-r \lambda_{2}(t)+w(t)\right) .
$$

Then $|v(t)| \leq \bar{v}$ for all $t \geq 0$ and

$$
\dot{\lambda}_{2}=s a t_{U_{2}}\left(-q \lambda_{2}(t)+v(t)\right) .
$$

We deduce that for any $\varepsilon>0$, there is $t_{\varepsilon} \geq 0$ such that for all $t \geq t_{\varepsilon}$

$$
\left|\lambda_{2}(t)\right| \leq(1+\varepsilon) \frac{\bar{v}}{q}
$$


Consequently,

$$
\left|-q \lambda_{2}(t)+v(t)\right| \leq(2+\varepsilon) \bar{v} .
$$

From the first inequality in (32), it follows that there is $\varepsilon_{p}>0$ such that $\left(2+\varepsilon_{p}\right) \bar{v}<U_{2}$ and $\left(1+\varepsilon_{p}\right) \frac{\bar{v}}{q}<U_{1}$. Then it follows from (38) and (39) that, for all $t \geq t_{\varepsilon_{p}}$,

$$
\left|\lambda_{2}(t)\right|<U_{1}
$$

and

$$
\left|-q \lambda_{2}(t)+v(t)\right|<U_{2}
$$

As an immediate consequence, we have that, for all $t \geq t_{\varepsilon_{p}}$,

$$
\left\{\begin{array}{l}
\dot{\lambda}_{1}=\lambda_{2} \\
\dot{\lambda}_{2}=-q \lambda_{2}+\operatorname{sat}_{\bar{v}}\left(-r q \lambda_{1}-r \lambda_{2}+w\right)
\end{array}\right.
$$

Now, using $\lambda_{3}=\lambda_{2}+q \lambda_{1}$, we have, for all $t \geq t_{\varepsilon_{p}}$,

$$
\left\{\begin{aligned}
\dot{\lambda}_{1} & =-q \lambda_{1}+\lambda_{3} \\
\dot{\lambda}_{3} & =-q \lambda_{2}+s a t_{\bar{v}}\left(-r q \lambda_{1}-r \lambda_{2}+w\right)+q\left(-q \lambda_{1}+\lambda_{3}\right) \\
& =s a t_{\bar{v}}\left(-r q \lambda_{1}-r \lambda_{2}+w\right) .
\end{aligned}\right.
$$

Finally,

$$
\left\{\begin{array}{l}
\dot{\lambda}_{1}=-q \lambda_{1}+\lambda_{3} \\
\dot{\lambda}_{3}=\text { sat }_{\bar{v}}\left(-r \lambda_{3}+w\right) .
\end{array}\right.
$$

Since $|w(t)| \leq \bar{w}$ for all $t \geq 0$, we deduce that for all $\delta>0$, there is $T_{\delta} \geq t_{\varepsilon_{p}}$ such that, for all $t \geq T_{\delta}$,

$$
\left|\lambda_{3}(t)\right| \leq \frac{(1+\delta) \bar{w}}{r} .
$$

As an immediate consequence,

$$
\left|-r \lambda_{3}(t)+w(t)\right| \leq r \frac{(1+\delta) \bar{w}}{r}+\bar{w}=(2+\delta) \bar{w} \leq(2+\delta) \frac{\bar{v}}{3},
$$

where the last inequality is a consequence of the second inequality in (32). Choosing $\delta=\frac{1}{2}$, we obtain

$$
\left|-r \lambda_{3}(t)+w(t)\right| \leq \bar{v},
$$

for all $t \geq T_{\frac{1}{2}}$. It follows that for all $t \geq T_{\frac{1}{2}}$, (35) is satisfied.

\section{MAIN RESUlT}

We are ready to state and prove the main result of the paper.

Theorem 1: Let $\phi$ and $\tau$ be respectively a function and a constant chosen as in Lemma 1 with $\bar{d}=2 \phi_{c} \bar{\delta}_{1}+2 \bar{\delta}_{2}$. Consider system (2) in closed loop with

$$
u\left(Y_{t}\right)=-q y_{3}(t)+\operatorname{sat}_{\bar{v}}\left(-r q y_{2}(t)-r y_{3}(t)+\varsigma\left(y_{1 t}\right)\right)
$$

with $Y=\left(y_{1}, y_{2}, y_{3}\right)$,

$$
\begin{aligned}
\varsigma\left(y_{1 t}\right)= & -\mathfrak{s} \phi\left(y_{1}(t)\right)+\mathfrak{s}\left(e^{-r \tau}+e^{-q \tau}\right) \phi\left(y_{1}(t-\tau)\right) \\
& -\mathfrak{s} e^{-(q+r) \tau} \phi\left(y_{1}(t-2 \tau)\right),
\end{aligned}
$$

with $\mathfrak{s}$ defined in (7) and where $r>0, q>0, \bar{v}$ and $\bar{\phi}$ are constants such that

$$
\bar{v}<\min \left\{\frac{L_{3}}{2}, q L_{2}\right\} \quad, \quad \bar{v} \leq 2 r L_{1}
$$

and

$$
\left[1+\left(e^{-r \tau}+e^{-q \tau}\right)+e^{-(q+r) \tau}\right] \bar{\phi} \leq \frac{\bar{v}}{6 \mathfrak{s}} .
$$

Then, when

$$
\bar{\delta}_{2} \leq \frac{\bar{v}}{6 q r},
$$

there is $t_{\star} \geq 0$ such that for all $t \geq t_{\star}, \quad X(t)=$ $\left(x_{1}(t), x_{2}(t), x_{3}(t)\right)$

$$
|X(t)| \leq \sqrt{\psi\left(\bar{\delta}_{1}, \bar{\delta}_{2}\right)}
$$

with

$$
\begin{aligned}
\psi(a, b)= & \bar{x}_{1}^{2}(a, b)+\left[\frac{10}{9} q b+\phi_{c} \bar{\eta} \bar{x}_{1}(a, b)\right]^{2} \\
& +q^{2}(1+q)^{2}\left[\frac{10 b}{9}+\frac{q\left(1+e^{-q \tau}\right) \phi_{c}}{1-e^{-q \tau}} \bar{x}_{1}(a, b)\right]^{2}, \\
\bar{x}_{1}(a, b)= & \frac{\sqrt{2}\left(2 \phi_{c} \bar{\eta} \tau+1\right)\left(\phi_{c} a+b\right)}{\sqrt{\mathfrak{c}(\mathfrak{c}-\tau \mathfrak{p})}} .
\end{aligned}
$$

Remark. The key feature of $u\left(Y_{t}\right)$ defined in (48) is that it depends only on the measurements and has no distributed term. Also, it is worth noticing that the functions $\phi$ and $\eta$ do not need to be of class $C^{1}$, which is important from an applied point of view.

Proof. The proof is omitted.

\section{ILLUSTRATION}

\section{A. Scenario and numerical data}

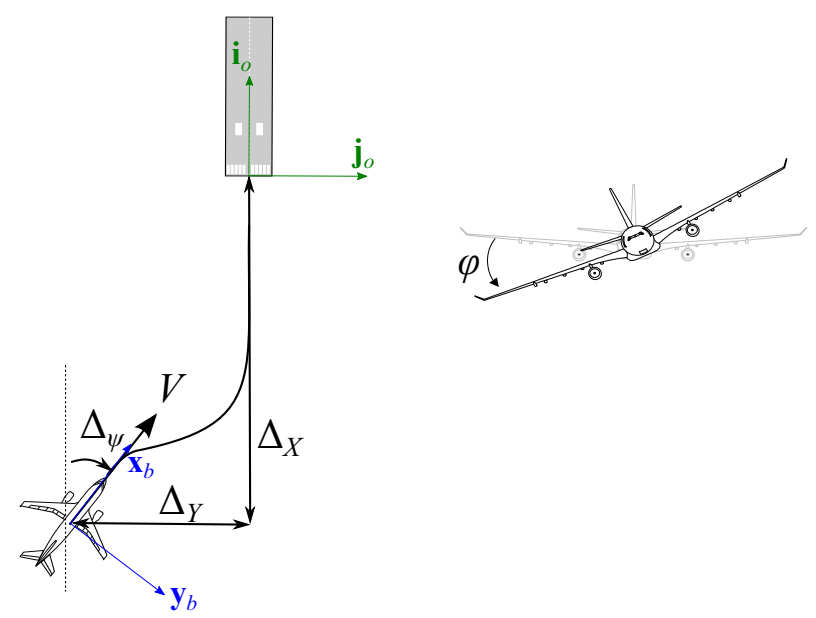

Fig. 1. Notations used in the alignment part of the glide phase

To illustrate our results, we consider the lateral dynamics of an Airbus airliner which must align with a runway using a body fixed monocular camera. This problem is a challenge of strong relevance in the case the runway is unequipped or in the case of GPS loss. More precisely, the position, size and heading of the runway are unknown, so that the relative position $\left(\Delta_{X}, \Delta_{Y}\right)$ and heading $\Delta_{\psi}$ of the aircraft with respect to it are unmeasured (Note that these quantities are depicted on Figure 1).

Airbus airliners safety is enhanced thanks to some flight envelope protections laws which can be automatically or 
fully automated [17]. For instance a protection, called high angle-of-attack prevents the aircraft from stalling [12]. Other protection laws are provided during lateral maneuvers: for instance, they can be designed

- either to limit the relative heading $\Delta_{\psi}$ between the aircraft and the runway (which is required to keep the runway in the field of view of the camera),

- or to limit the aircraft roll angle $\varphi$ and its time derivative $\dot{\varphi}$ (which is required to protect the aircraft from exceeding its operating limits).

All these protection laws directly act at the control surface deflection level. In case of control saturations or multiple active flight control laws, the aircraft response should be as fast as possible while respecting the highest priority constraint.

As discussed in [4], a key step for the successful transfer to the aeronautics sector of the developed control methods is their demonstration on standardized industrial V\&V processes. The control design methods are validated by using more and more complex aircraft models. Once the tuning process is satisfactory, the methods are finally applied to the nonlinear and most representative aircraft model.

The starting point of this research was thus a very simplified lateral guidance model provided by Airbus:

$$
\left\{\begin{aligned}
\dot{\Delta}_{Y} & =V \operatorname{sat}_{L_{\psi}}\left(\Delta_{\psi}\right) \\
\dot{\Delta}_{\psi} & =\frac{g}{V} \operatorname{sat}_{L_{\varphi}}(\varphi) \\
\dot{\varphi} & =\operatorname{sat}_{L_{u}}\left(u_{\text {lat }}\right)
\end{aligned}\right.
$$

where $u_{\text {lat }}$ is the input, where $V=70 \mathrm{~m} . \mathrm{s}^{-1}$ is constant all along the final approach, $g=9.81 m . s^{-2}$ and $\varphi$ (resp. $u_{\text {lat }}$ ) is the aircraft roll angle (resp. the guidance/outer loop control action).

After a simple change of coordinates, it is obvious that such a model belongs to the class of system (2). Clearly, this model does not have any physical interpretation nonetheless it is currently used by Airbus for its lateral guidance design studies: indeed, even if this model is very simplified, it allows the user to preliminary tune a guidance law while keeping in mind that the full model possesses some protection laws. As a result, using such a model should provide a first quantitative feedback of the quality of the guidance design.

In our context and using the extraction method proposed in [1], we also assume that the (possibly noisy) quantities $\frac{\Delta_{Y}}{\Delta_{X}}$ and $\Delta_{\psi}$ can be derived by image processing from the body fixed monocular camera at each instant. The unknown term $\Delta_{X}=\eta^{-1}$ is the longitudinal distance between the aircraft center of gravity and the runway touchdown point (as shown on Figure 1). In this application, we focus on the glide phase just before switching to the final flare phase. In this phase, the term $\Delta_{X}(t=0)$ belongs to $[4000 m, 6000 m]$ and evolves at a constant speed $\mathrm{V}$.

Moreover, it is assumed that $\varphi$ can be perfectly measured by using the aircraft IMU ${ }^{1}$.

It is easy to see that system (55) together with the measured outputs can be transformed into system (2)-(3)

\footnotetext{
${ }^{1}$ Inertial Measurement Unit
}

by applying the change of coordinates $x_{1}=\Delta_{Y}, x_{2}=V \Delta_{\psi}$, $x_{3}=g \varphi$ while changing the saturation limits accordingly and by multiplying the measured outputs by some known constants. The saturations limits are as follows:

$$
L_{1}=25 m \cdot s^{-1} \quad, \quad L_{2}=7 m \cdot s^{-2} \quad, \quad L_{3}=2 m \cdot s^{-3} .
$$

Note that they correspond to $L_{\psi} \cong 20 \mathrm{deg}, L_{\varphi} \cong 40 \mathrm{deg}$ and $L_{u} \cong 12 \mathrm{deg} / \mathrm{s}$.

\section{B. Controller gains tuning}

We first propose to use the measure $\frac{\Delta_{Y}}{\Delta_{X}}$ and to multiply it by $\hat{\Delta}_{X}=\hat{\Delta}_{X}(t=0)-V t$ where $\hat{\Delta}_{X}(t=0) \in[4500 m, 6000 m]$ is a tuning parameter (another possibility would be to design a vision based estimation of $\Delta_{X}$ following for instance the work of [5], [3]). We decided to choose the minimum value $\hat{\Delta}_{X}(t=0)=4500$ to obtain

$$
\bar{\eta}=1
$$

We thus used the following quantity in our control law:

$$
y_{1}=\eta(t) \Delta_{Y}=\frac{4500-V t}{\Delta_{X}} \Delta_{Y}
$$

the following parameters were chosen for the unconstrained control law in order to satisfy some industrial specifications:

$$
q=0.3, \quad r=0.59
$$

Also, for $\phi$ and $\tau$, we choose

$$
\phi(s)=0.15 \tanh (0.4 s)
$$

and $\tau=2 \mathrm{~s}$ in order to satisfy inequality (9) when $\bar{d}=0$. (Let us observe that we thus have $\bar{\phi}=0.15$ and $\left.\phi_{c}=0.06\right)$. The conditions of our main result are also satisfied choosing:

$$
\bar{v}=1.02 \text {. }
$$

\section{Numerical results}

Our design is applied on the aircraft benchmark which is a non-trivial application. Clearly this example goes beyond what is proved in the paper since simulations stop when $\Delta_{X}=400 m$ which corresponds to the beginning of the flare phase: this is a finite time problem and global stabilization results are lost. Interestingly, our control method succeeds to considerably lower the lateral deviation $\Delta_{Y}$ for a set of initial conditions $\delta_{X}(0) \in[4500 m, 6000 m], \Delta_{Y}(0) \in[-30 m, 30 m]$, $\Delta_{\psi} \in[-3 d e g, 3 d e g]$ as shown on Figure 2. As shown on Figure 3 , the method is still efficient when we the vision based outputs are noisy (we here chose $\delta_{1}(t)=4 \cdot 10^{-3} \hat{\Delta}_{X}(t)$ and $\delta_{2}=0.3 \mathrm{deg}$ ).

\section{Further discussion}

As shown on Figure 4, the numerical results are even better when multiplying $\phi$ by 2 . In this case, the conditions of our results are violated although the control law $u_{\text {lat }}$ is still far from its limitations. As a consequence, it may be possible to improve the control law performance. This issue is postponed to future studies. 

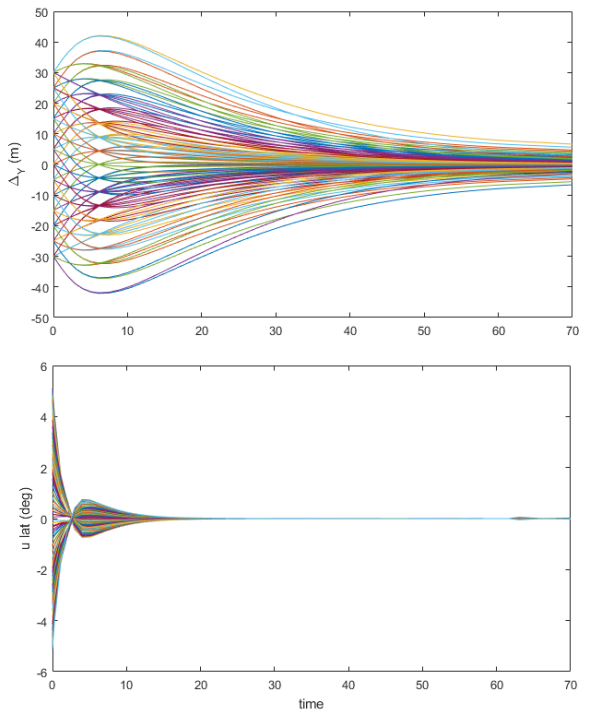

Fig. 2. Evolution of $\Delta_{Y}(t)$ and $u_{\text {lat }(t)}$ (below)
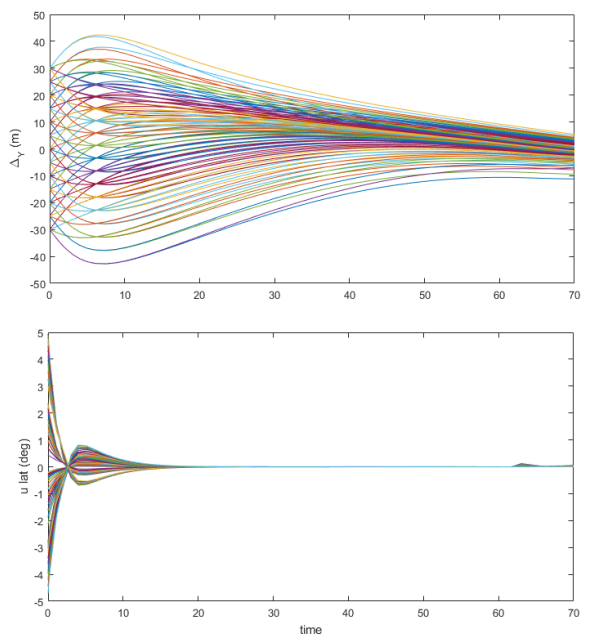

Fig. 3. Evolution of $\Delta_{Y}(t)$ and $u_{\text {lat }(t)}$ (below) in presence of outputs noise

\section{CONCLUSION}

We adapted a recent backstepping strategy to the problem of stabilizing a class of nonlinear systems when the output measurements are imprecise. Our design was illustrated on the lateral dynamics of a vision based landing aircraft problem. Ongoing work is devoted to extend our design on a more representative and complex aircraft model. Such a design will finally be validated in simulation on an industrial platform including image processing algorithms on synthetic images.

\section{REFERENCES}

[1] L. Burlion, H. de Plinval, Vision based anti-windup design with application to the landing of an Airliner, 20th IFAC World Congress, July, Toulouse, France, 2017.

[2] R. Freeman, L. Praly, Integrators backstepping for bounded controls and control rates, IEEE Trans. Automat. Control, 43 (1998), pp. 258262.
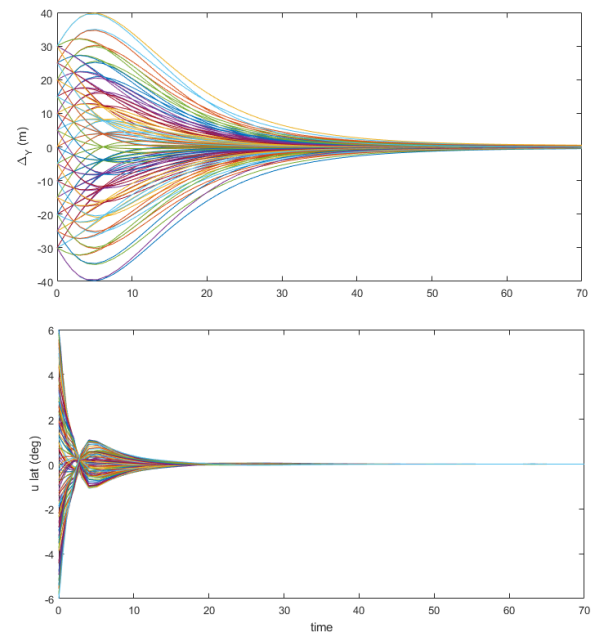

Fig. 4. Evolution of $\Delta_{Y}(t)$ and $u_{\text {lat }(t)}$ (below) when $\phi$ is multiplied by 2

[3] V. Gibert, L. Burlion, A. Chriette, J.Boada, F. Plestan, Nonlinear observers in vision system: Application to civil aircraft landing, European Control Conference, pp. 1818-1823, Linz, Austria, 2015.

[4] P. Goupil, J. Boada-Bauxell, A. Marcos, E. Cortet, M. Kerr and H. Costa, AIRBUS efforts towards advanced real-time Fault Diagnosis and Fault Tolerant Control, 19th IFAC World Congress, pp. 34713476, Cape Town, South Africa, Dec. 2014.

[5] D. Karagiannis, A. Astolfi, A new solution to the problem of range identification in perspective vision systems, IEEE Trans. Automat. Control, 50 (12) : 2074-2077, 2005.

[6] M. Malisoff, F. Mazenc, Constructions of Strict Lyapunov Functions. Springer-Verlag. London, 2009.

[7] F. Mazenc, A. Iggidr. Backstepping with Bounded Feedbacks. Systems and Control Letters, Vol. 51/3-4, pp. 235-245, 2004.

[8] F. Mazenc, M. Malisoff, New Control Design for Bounded Backstepping under Input Delay. Automatica, vol. 66, pp. 48-55, April 2016.

[9] F. Mazenc, M. Malisoff, J. Weston, New bounded backstepping control designs for time-varying systems under converging input converging state conditions. 55th IEEE Conference on Decision and Control, pp. 3167-3171, Las Vegas, NV, USA, Dec. 2016.

[10] F. Mazenc, S. Mondié, S.I. Niculescu. Global Asymptotic Stabilization for Chains of Integrators with a Delay in the Input. IEEE Trans. Automat. Control, Vol. 48, no.1, pp. 57-63, 2003.

[11] F. Mazenc, L. Praly. Adding an Integration and Global Asymptotic Stabilization of Feedforward Systems. IEEE Trans. Automat. Control, Vol. 41, no.11, pp.1559-1578, 1996.

[12] S. Oudin, S. Delannoy and P. Debusscherre, Low Speed Protections for a Commercial Airliner: a Practical Approach, The AIAA Guidance, Navigation, and Control Conference, AIAA SciTech Forum, 2017.

[13] A. Saberi, Z. Lin, A.R. Teel, Control of linear systems with saturating actuators, IEEE Trans. Automat. Control, Vol. 41, no. 3, pp. 368-378, 1996.

[14] R. Sepulchre, M. Jankovic, P. V. Kokotovic, Constructive Nonlinear Control, Springer-Verlag, Berlin, 1996.

[15] S. Tarbouriech, G., Garcia, J.M.G., da Silva Jr, I., Queinnec, Stability and stabilization of linear systems with saturating actuators. SpringerVerlag, Berlin, 2011.

[16] A.R. Teel, Feedback stabilization: Nonlinear solutions to inherently nonlinear problems. Memorandum no. UCBERL M92/65, June 12, 1992.

[17] P. Traverse, I. Lacaze, J. Souyris, Airbus fly-by-wire: A total approach to dependability, 18th IFIP World Computer Congress, pp. 191-212, Toulouse, France, 2004. 